\title{
OBSERVATIONAL ASTEROSEISMOLOGY OF $\delta$ SCUTI STARS
}

\author{
BREGER M. AND AUDARD N. \\ Institut of Astronomy, University of Vienna \\ Türkenschanzstraße 17, A-1180 Wien Austria
}

\begin{abstract}
Ground-based observational networks are now able to reliably detect more than 20 frequencies for individual $\delta$ Scuti stars. The groundbased Delta Scuti Network specializes in the intensive study of individual stars for several months with dedicated telescopes on different continents. With the new quantity and quality of data, the observational limit to extracting 50 or more frequencies in a single $\delta$ Scuti star is no longer determined only by the signal/noise ratio, but by the available frequency resolution.
\end{abstract}

Recent results on the $\delta$ Scuti stars FG Vir and $4 \mathrm{CVn}$ are presented. $4 \mathrm{CVn}$ exhibits some of the characteristics of $\delta$ Scuti stars: some close spacings are observed, pulsations occur in specific frequency regions with relatively sharp borders, and show large amplitude variations. This presents an important asteroseismological diagnostic, and can help us to improve our understanding of for example rotation and excitation mechanisms.

We also report a new Gamma Doradus variable with two excited $g$ modes (HD 108100, F2V).

\section{Introduction}

The $14^{\text {th }}$ DSN campaign was devoted to the $\delta$ Scuti star FG Vir. Observations were carried out in a combined photomultiplier campaign by DSN and WET (Whole Earth Telescope) networks. Furthermore, about one month of CCD data was collected. Two observational methods are used, depending on the observed frequency-range: for periods larger than about 30 minutes, the single-channel 3-star technique is adopted, while for periods smaller than 30 minutes, the high-speed photometry technique (one channel measurements on the target all the time) is adopted. The program PERIOD 
(Breger, 1990b) is used to detect the frequencies in the photometric data. These new observations confirmed all previously certain peaks, as well as many peaks, which were previously not found to be statistically significant. The data are currently being reduced, and the latest results reveal 23 frequencies. This is one of the largest numbers of detected frequencies for a single $\delta$ Scuti star. The presence of some gravity modes has already been reported by Breger et al. (1995) and Guzik and Bradley (1995). Some more $\mathrm{g}$ modes, with possible regular spacing, might have been detected in these new observations. These modes are particularly interesting for improving our knowledge of rotation (Goupil et al, 1996) and of processes such as convective-core overshooting (Dziembowski and Pamyatnykh 1991, Audard et al 1995).

The $\delta$ Scuti 4 CVn was observed within the $15^{\text {th }}$ DSN campaign, by Breger, Handler, Garrido, Audard, Beichbuchner, Zima, Paparo, Li Zhiping, Jiang Shi-yang, Liu Zong-li, Zhou Ai-ying, Pikall, Stankov, Guzik, Sperl, Kresinski, Ogloza, Pajdosz, Zola, Serkowitsch, Reegen, Rumpf, Solheim, Schmalwieser and Thomasson. 25 frequencies have been extracted in a preliminary analysis, among which we also find frequency combinations.

\section{Specific frequency regions and close spacing: $4 \mathrm{CVn}$}

A summary of the results for $4 \mathrm{CVn}$ is represented Fig. 1. The pulsation occurs in a specific frequency region, between roughly 5 and 10 cycles/day, and the peaks at lower and higher period are frequency combinations. These features do not appear in FG Vir. The main frequencies have a dual nature, i.e. they are $\mathrm{g}$ modes in the deep stellar interior and p modes in the outer parts. Dziembowski (1997) states that they are g-like modes of high radial order in the deep regions.

Another property of some $\delta$ Scuti stars is the close spacing observed for some pulsations. In the case of FG Vir, two modes are separated by as little as $0.03 \mathrm{c} / \mathrm{d}(0.3 \mu \mathrm{Hz})$. This spacing is too small to be due to rotational splitting, as found by for example Michel et al. (1996), Goupil et al. (1996), Pérez-Hernández et al. (1995). The pulsations can thus only correspond to modes of different degrees.

\section{Amplitude variations}

From 1966 to 1996, the amplitude of the pulsations of 4CVn varies drastically. Breger (1990a) states that this is more likely due to the Blazhko effect, rather than a beating phenomenom between two close frequencies.

We do not yet clearly know why the amplitudes vary, neither why only a small fraction of theoretically unstable modes are effectively observed. Some predictions are made for radial modes by e.g. Houdek et al. (1995). 


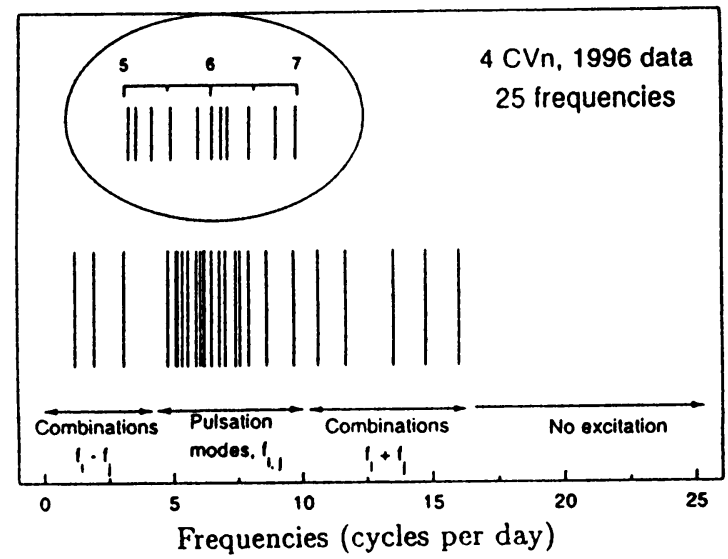

Figure 1. Observed pulsation spectrum of $4 \mathrm{CVn}$ (preliminary). The inset shows the peaks between 5 and 7 cycles/day.

The nonlinearity problem for radial and nonradial modes is addressed by Buchler et al. (1995), Goupil and Buchler (1994).

These varying amplitudes are particularly powerful for our understanding of the excitation mechanism, and of growth and decay rates.

\section{4. $\gamma$ Doradus stars}

The variability of $\gamma$ Doradus stars has been discovered only recently. These stars are early $\mathrm{F}$ stars, or are located just above the main sequence. They lie near the cool border of the instability strip of $\delta$ Scuti stars. They pulsate at very low frequencies, with a typical value near $1 \mathrm{c} / \mathrm{d}$. The corresponding values of the pulsational constant, $Q$, are about ten times the value for the fundamental radial mode. Therefore, the pulsations can only be identified as high-order g modes. $\gamma$ Doradus itself has been observed for example by Balona et al. 1996. Aerts and Krisciunas (1995) report results of 9 Aur. Breger and Beichbuchner (1996) give a general review.

Breger et al. (1996) have recently confirmed the variability of the $\gamma$ Dor star HD 108100, within a coordinated campaign with the DSN and WET networks. Two pulsations of periods 17.1 and 18.1 hours have been detected (see Fig. 2).

At the present time, $\gamma$ Doradus stars are a challenge to asteroseismology. The theoretical frequency spectrum of $g$ modes is very dense, and an unambiguous mode identification is almost impossible. It is moreover made even more difficult by the effects of rotation. Therefore asteroseismology of $\gamma$ Doradus stars is still waiting for accurate models with high-order $g$ 


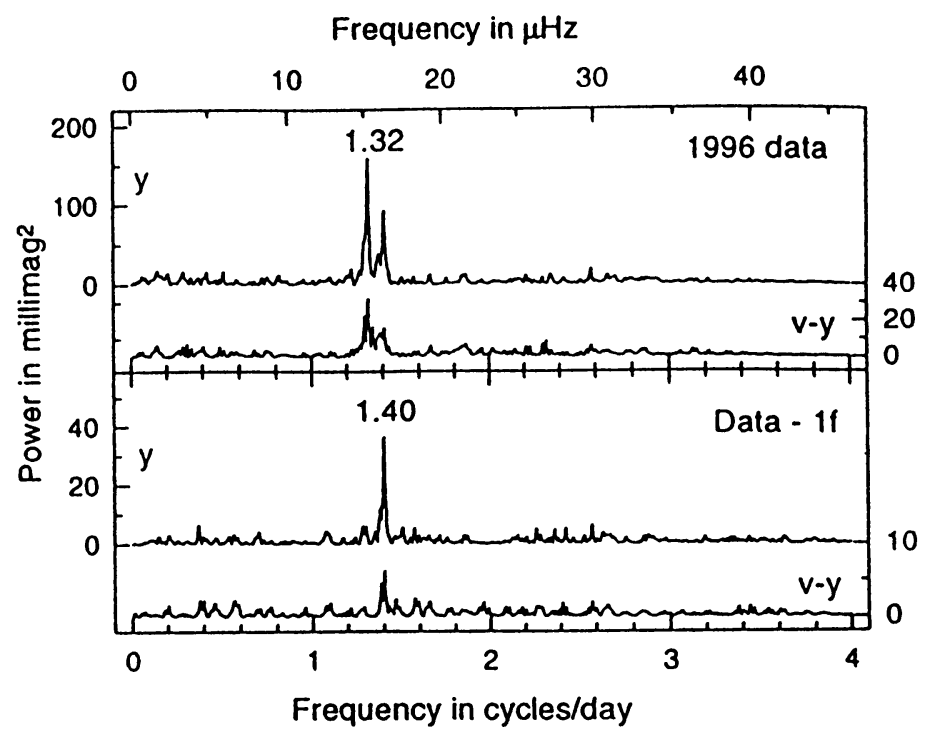

Figure 2. Power spectrum of the new $\gamma$ Doradus star HD 108100

modes.

\section{Steps towards mode identification}

\subsection{FIRST MODE IDENTIFICATION}

A first mode identification can be performed by determining the phase differences between $y$ and $v$ light curves. Garrido et al. (1990) have shown that these can be used reliably to discriminate between radial and nonradial modes of low radial order and low degree.

\subsection{COMPARISON BETWEEN OBSERVATIONS AND THEORETICAL MODELS}

Guzik and Bradley (1995) have computed models for FG Vir, and compared their results to those from Breger et al. (1995), obtained from Dziembowski's evolution and pulsation codes. The slight differences between Dziembowski's codes, and Guzik and Bradley's, result in nonradial frequencies which can differ by several $\mu \mathrm{Hz}$. As a result, Guzik and Bradley identified pulsations with the same radial orders and degrees $(n, \ell)$ as Breger et al. (1995), but with different azimuthal orders $m$. Moreover, they find that only models of 1.8 and $1.82 M_{\odot}$ can match the 10 first detected frequencies of FG Vir found during the 1995 FG Vir campaign, and no model can match 
all the 19 frequencies. Bradley and Guzik (1996) also report the effects of the OPAL95 and OPAL92 opacities, and of different equations of state, on the mode identification of the frequencies of FG Vir and the $\delta$ Scuti CD-24 7599 .

This lack of uniqueness of models reveals the inaccuracies of parts of the input physics and of the description of phenomena such as rotation and convective core overshooting. Another source of uncertainty is our knowledge of the basic fundamental parameters of the stars, such as mass and effective temperature. The uncertainties are often too large to constrain the models, so that several models can match the same star in the HR diagram.

The observed frequencies of pulsation can be used to correct deficiencies in the structure and the input physics of the evolution and oscillation codes (opacities, equation of state, rotation, overshooting,...), if one or both of the following two conditions are fulfilled: 1) modes are identified by amplitude and/or phase studies of colours or spectroscopic data, 2) precise stellar parameters are known for stars, where special techniques can be employed to obtain these parameters (binaries, open cluster stars, very nearby stars).

\section{Conclusion}

We need more frequency resolution to resolve the peaks of the power spectra. Spectroscopic data of best quality are required to provide more constraints to the modelling. These can only be obtained with longer, multisite campaigns.

Stellar models have to be improved, with a better description of processes such as rotation and convective-core overshooting.

With more than 20 frequencies for a single star, asteroseismology of $\delta$ Scuti stars is a powerful tool for improving our knowledge of the interior and evolution of stars.

\section{References}

Aerts C., Krisciunas K. (1996) MNRAS, 278, 877

Audard N., Provost J., Christensen-Dalsgaard J. (1995) Astron. Astrophys., 297, 427

Balona L.A., Hearnshaw J.B., Koen C., Collier A., Machi I., Mkhosi M., Steenberg C. (1994) MNRAS, 267, 103

Bradley P.A., Guzik J.A. (1996) in Sounding solar and Stellar Interiors, poster book

Breger M., Beichbuchner F. (1996) Astron. Astrophys., 313, 851

Breger M., Handler G., Garrido R., Audard N., Beichbuchner F., Zima W., Paparo M., Li Zhi-ping, Jiang Shi-yang, Liu Zong-li, Zhou Ai-ying, Pikall H., Stankov A., Guzik J.A., Sperl M., Kresinski J., Ogloza W., Pajdosz G., Zola S., Serkowitsch E., Reegen P., Rumpf T., Schmalwieser A. (1996) Astron. Astrophys., in press

Breger M., Handler G., Nather R.E., Winget D.E., Kleinman S.J., Sullivan D.J., Li Zhiping, Solheim J.E., Jiang Shi-yang, Liu Zong-li, Wood M., Watson T.K., Dziembowski W.A., Serkowitsch E., Mendelson H., Clemens C., Krzesinski J., Pajdosz G. (1995) Astron. Astrophys., 297, 473 
Breger M. (1990a) Astron. Astrophys., 240, 308

Breger M. (1990b) Comm. Asteroseismology (Vienna) 20, 1

Buchler J.R., Goupil M.J., Serre T. (1995) Astron. Astrophys., 296, 405

Dziembowski W.A. (1997) these proceedings

Dziembowski W.A., Pamyatnkh A.A. (1991) Astron. Astrophys., 248, L11

Garrido R., Garcia-Lobo E., Rodriguez E. (1990) Astron. Astrophys., 234, 262

Goupil M.J., Buchler J.R. (1994) Astron. Astrophys., 291, 481

Goupil M.J., Dziembowski W.A., Goode P.R., Michel E. (1996) Astron. Astrophys., 305, 487

Guzik J.A., Bradley P.A. (1995) $\delta$ Scuti Newsletter, issue 9

Houdek G., Rogl J., Balmforth N., Christensen-Dalsgaard J. (1995) in GONG'94: Helioand Astero-seismology from Earth and Space, Ulrich R.K., Rhodes Jr E.J., Däppen W. (eds.), PASPC 76, p. 641.

Michel E., Chevreton M., Goupil M.J., Belmonte J.A., Jiang S.Y., Alvarez M., Suran M., Soufi F., Auvergne M., Baglin A., Liu Y.Y., Vidal I., Hernandez M., Fu N. (1996) in Fourth SOHO Workshop: helioseismology, p.543.

Pérez Hernández F., Claret A., Belmonte J.A. (1995), Astron. Astrophys., 295, 113 